\title{
Insight to shape of soil microbiome during the ternary cropping system of Gastradia elata
}

Qing-Song Yuan ${ }^{1 \dagger}$, Jiao Xu ${ }^{1 \dagger}$, Weike Jiang ${ }^{1}$, Xiaohong Ou' ${ }^{1}$, Hui Wang ${ }^{1}$, Lanping Guo ${ }^{2}$, Chenghong Xiao ${ }^{1}$, Yanhong Wang ${ }^{1}$, Xiao Wang ${ }^{3}$, Chuanzhi Kang ${ }^{2}$ and Tao Zhou ${ }^{1 *}$

\begin{abstract}
Background: The ternary cropping system of Gastradia elata depends on a symbiotic relationship with the mycorrhizal fungi Armillaria mellea, which decays wood to assimilate nutrition for the growth of G. elata. The composition of microbe flora as key determinants of rhizoshere and mycorrhizoshere soil fertility and health was investigated to understand how G. elata and A. mellea impacted on its composition. The next generation pyrosequencing analysis was applied to assess the shift of structure of microbial community in rhizoshere of $G$. elata and mycorrhizoshere of A. mellea compared to the control sample under agriculture process.

Results: The root-associated microbe floras were significantly impacted by rhizocompartments (including rhizoshere and mycorrhizoshere) and agriculture process. Cropping process of G. elata enhanced the richness and diversity of the microbial community in rhizoshere and mycorrhizoshere soil. Furthermore, planting process of $G$. elata significantly reduced the abundance of phyla Basidiomycota, Firmicutes and Actinobacteria, while increased the abundance of phyla Ascomycota, Chloroflexi, Proteobacteria, Planctomycetes, and Gemmatimonadetes in rhizoshere and mycorrhizoshere. Besides, A. mellea and G. elata significantly enriched several members of saprophytoic and pathogenic fungus (i.e., Exophiala, Leptodontidium, Cosmospora, Cercophora, Metarhizium, Ilyonectria, and Sporothrix), which will enhance the possibility of G. elata disease incidence. At the same time, the ternary cropping system significantly deterred several members of beneficial ectomycorrhizal fungus (i.e., Russula, Sebacina, and Amanita), which will reduce the ability to protect G. elata from diseases.
\end{abstract}

Conclusions: In the ternary cropping system of G. elata, A. mellea and G. elata lead to imbalance of microbial community in rhizoshere and mycorrhizoshere soil, suggested that further studies on maintaining the balance of microbial community in A. mellea mycorrhizosphere and G. elata rhizosphere soil under field conditions may provide a promising avenue for high yield and high quality G. elata.

Keywords: Gastradia elata, Armillaria mella, Microbiome, Rhizosphere soil, Mycorrhizosphere soil, Ternary cropping system, Next generation pyrosequencing

\footnotetext{
*Correspondence: taozhou88@163.com

${ }^{\dagger}$ Qing-Song Yuan and Jiao Xu contributed equally to this work.

${ }^{1}$ Guizhou University of Traditional Chinese Medicine, Dongqingnan Road, Guiyang 540025, Guizhou, China

Full list of author information is available at the end of the article
}

(C) The Author(s). 2020 Open Access This article is licensed under a Creative Commons Attribution 4.0 International License, which permits use, sharing, adaptation, distribution and reproduction in any medium or format, as long as you give appropriate credit to the original author(s) and the source, provide a link to the Creative Commons licence, and indicate if changes were made. The images or other third party material in this article are included in the article's Creative Commons. licence, unless indicated otherwise in a credit line to the material. If material is not included in the article's Creative Commons licence and your intended use is not permitted by statutory regulation or exceeds the permitted use, you will need to obtain permission directly from the copyright holder. To view a copy of this licence, visit http://creativecommons.org/licenses/by/4.0/ The Creative Commons Public Domain Dedication waiver (http://creativecommons.org/publicdomain/zero/1.0/) applies to the data made available in this article, unless otherwise stated in a credit line to the data. 


\section{Background}

Rhizosphere soil is an important compartment direct contact with plants root or mycorrhiza rhizomorph, which play critica roles to assimilate nutrients, resist stress, and combat disease. Furthermore, rhizosphere microbes are key determinants of soil fertility and health. Imbalance of soil microbes have been identified as the most important factor contributing to elevated disease incidence in plants $[1,2]$. For examples, many researchers reported that the imbalance of microbial community in soil is the main reason for consecutive monoculture problem of many medicinal plants, such as Pseudostellaria heterophylla [3, 4], Rehmannia glutinous $[5,6]$. What's more, soil microbes have great influence on soil biogeochemical processes including decomposition, mineralization, and retention of soil nutrients [7]. Conversely, plants also can significantly affect the composition of soil microorganisms, change soil physical and chemical properties during agricultural operations through secret phytochemicals [8]. For example, Haichar el al's researches demonstrated that plant-derived phytochemicals secreted by root are able to structure the rhizosphere microbiome by deterring or attracting certain microbial species [9]. And also Radix notoginseng eventually caused a shift in soil microbes from a bacterial-dominant community to a fungal-dominant community through increase $\mathrm{N}$ application rates in its agricultural ecosystems [10].

Microbial community structure in soil is very complicated, in which the microbes in soil commonly includes bacteria, fungi, protozoa, etc. Alternation of microbial composition, especially the structure change between bacteria and fungi,can make plants susceptible to diseases and poor growth. The main reason of the altenation of microbial composition in soil is the interactions between bacteria, fungi, such as the antagonistic effect of probiotics and pathogenic fungi, which plays crucial roles in maintaining the balance of microbial community in soil $[11,12]$. To date, in tuberous medicinal plant, numerous studies have shown that plant root rot complex are main caused by the increase of the abundance of pathogenic fungus including Fusarium spp. [13, 14], Rhizoctonia solani [15], Alternaria spp. [16, 17], Phytophthora sojae [18] in soil. Many biocontrol researches managing crops disease demonstrated that antagonism bacteria, for example Bacillus amyloliquefaciens S76-3 as soil amendment and improvement, have a good application to manage the disease of various crops [19-21]. Additionally, $\mathrm{Wu}$ et al. pointed out that the relative abundances of Fusarium, Cylindrocarpon, Rhizoctonia, etc. remarkably increased after agriculture process of Rehmannia glutinosa, as well the relative abundance of beneficial bacterial Pseudomonas aeruginosa decreased after agriculture process of Rehmannia glutinosa [5].
Therefore, It is very important for G. elata production to determine the alternation of bacteria and fungi community in its ternary cropping system.

As a perennial herbaceous plant, G. elata belongs to the achlorophyllous orchid and is widely distributed in Asian. G. elata is highly valued in medicine, health care and food, primarily due to the various active compounds of G. elata, such as gastrodin, polysaccharide, organic acid, etc. [22-24]. The main producing areas of G. elata mainly contain the eastern Asian countries such as Chinese, Southern Korea, Japanese, etc. Especially in china, the cultivation of G. elata is the most common. The artificial cultivation system of G. elata is very special, in which the mycorrhizal fungi $A$. mellea decomposes litter and trees, assimilates nutrition to promote the growth of $G$. elata $[25,26]$. And more, previously studies showed that a number of crops could reshape the microbiome of rhizosphere soil under agriculture process, such as potato [27], soybean [1], wheat [2], and banana [28], as well as Chinese medicine herbs such as Pseudostellaria heterophylla [3, 4], Rehmannia glutinous $[5,6]$. But at present, there is no report on whether $G$. elata and $A$. mellea have impact on the community structure and the abundance of the microflora in rhizosphere or mycorrhizoshere soil under agriculture process.

Besides, early studies have dominated that A. mellea, as a medicine fungi could produce many antibacterial and antifungal compounds, such as sesquiterpene aryl esters and armillaric acid, which exhibits high inhibitory activity against gram-positive bacteria, yeast, Streptococcus spp., Mucor spp., Trichoderma spp., Rifai aggr., Rhizopus stoloniferp, Fusarium spp., and Gliocladium viren [29-32]. What's more, A. mellea possess strongly antifungal activity because it have the ability to induce $G$. elata to produce a set of defense proteins [33, 34]. But, it is not clear how G. elata and A. mellea affect the microbial community in the rhizosphere soil in the ternary cropping system. Therefore, we conducted G. elata planting experiments on the G. elata geo-authentic producing area Dafang County in China. Then, we collected the (mycor) rhizsphere soils after agriculture process and used the next generation sequencing technology of ITS and V3V4 rDNA amplicons in Illumina MiSeq platform to characterize microbial communities in rhizosphere and mycorrhizosphere soils and assess how G. elata and $A$. mellea shape the soil microbial community structure after $G$. elata cropping process.

\section{Results}

General statistic of reads and OTUs in sequencing data

The next generation sequencing (NGS) of V3V4 and ITS rDNA amplicons was applied to assess the changes in the rhizosphere and mycorrhizosphere microbial 
communities after G. elata cropping process. After removal of short and low-quality reads, singletons, and chimeras, 19,555 from 34,971 effective sequences were obtained for V3V4 rDNA amplicons, as well as 48,999 from 67,295 effective sequences were obtained for ITS rDNA amplicons in total, and the detailed information of sequencing data in each sample was displayed in supplementary Table S1 and S2. Based on 97\% similarity, $1710,1762,1669$, and 1148 OUTs were obtained for bacterial community in the rhizoshere soil samples from the mature tube of G. elata (GE1), rhizomorph of A. mellea (A1), the mother tube of G. elata containing rhizomorph of A. mellea (GEA) and no planted (control), respectively. As well as based on $97 \%$ similarity 1367, 1348, 1418 , and 671 OTUs were obtained for fungal community in GE1, A1, GEA, and control, respectively (Fig. 1).

\section{Alpha diversity analysis in different Rhizoshere soils}

The microbiota diversity and richness of different rhizoshpere soil samples were investigated by Ace, Chao, Shannon indexes based on ITS and V3V4 sequence data. In bacteria community, A1 showed the highest diversity and highest species richness with the observed species, Chao, Ace indices at 1762, 1131.2, 1157.2, respectively (Fig. 2). Control had the lowest species richness and diversity compared with the other three rhizosphere soil groups (Fig. 2). All indices of richness and diversity demonstrated AGE and GE were significantly different from the control, but no significant difference was observed between AGE and GE. In fungi community, the richness and diversity of A1 were lower than AGE and GE, but higher than control (Fig. 2 D, E, F). Similarly, the simposon indices had the same results (Fig. S1). The results showed that G. elata and A. mellea could elevate the richness and diversity in rhizoshere and mycorrhizoshere soil, and A. mellea had the lower effect on the fungi community than the bacteria community in mycorrhizoshere soil.

Microbiota diversity in different Rhizoshere soils under G. elata cropping process

In order to investigate the alteration patterns of microbiota in the different rhizoshere soils, the beta diversity patterns were estimated by the principal coordinate

\section{A}

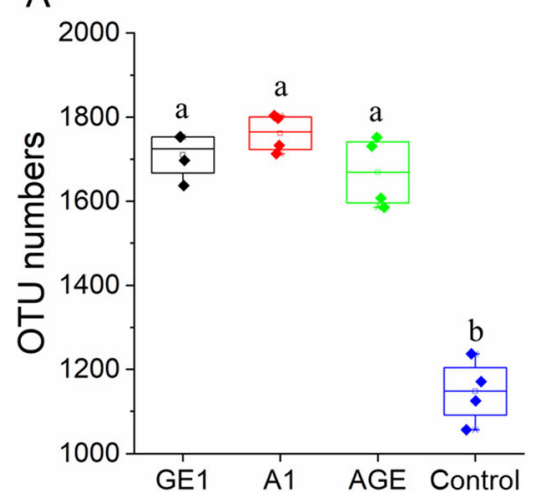

C

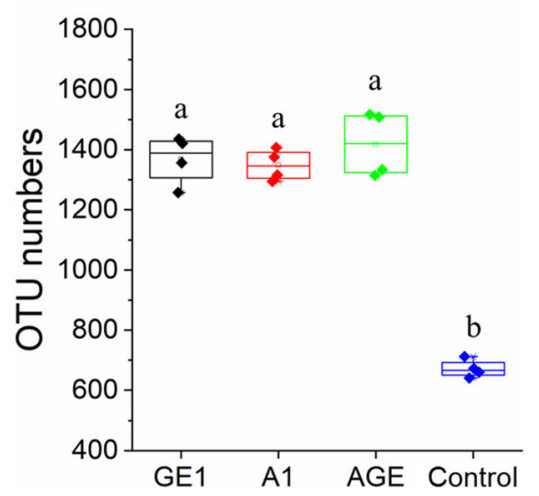

B

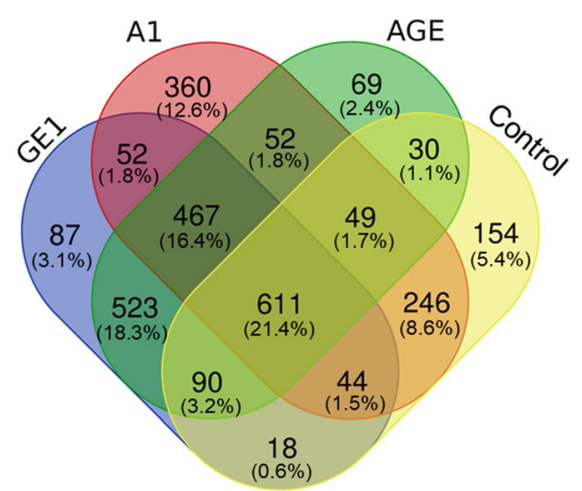

$\mathrm{D}$

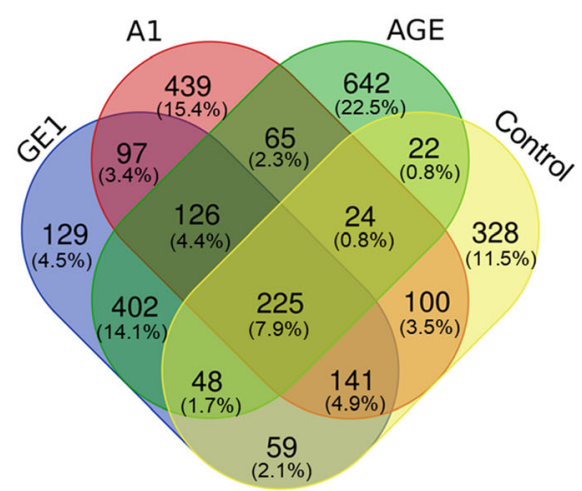

Fig. 1 OTU numbers and venn analysis exclusive and shared OTUs in different rhizoshere and mycorrhizoshere soil samples. $\mathbf{A}$ the numbers of OTU bacterial community; B the venn diagram in bacterial community; $\mathbf{C}$ the numbers of OTU fungal community; $\mathbf{D}$ the venn diagram in fungal community. GE1, A1, AGE, and control represent the rhizoshere or mycorrhizoshere soil of G. elata tubers, A. mellea rhizomorphs, G. elata tubers with $A$. mellea rhizomorphs, and unplanted, respectively. Lowercases $a$ and $b$ in figures show significantly differences between the different rhizocompartment soils (GE1, A1, AGE and control), determined by Tukey's test $(p<0.05)$ 

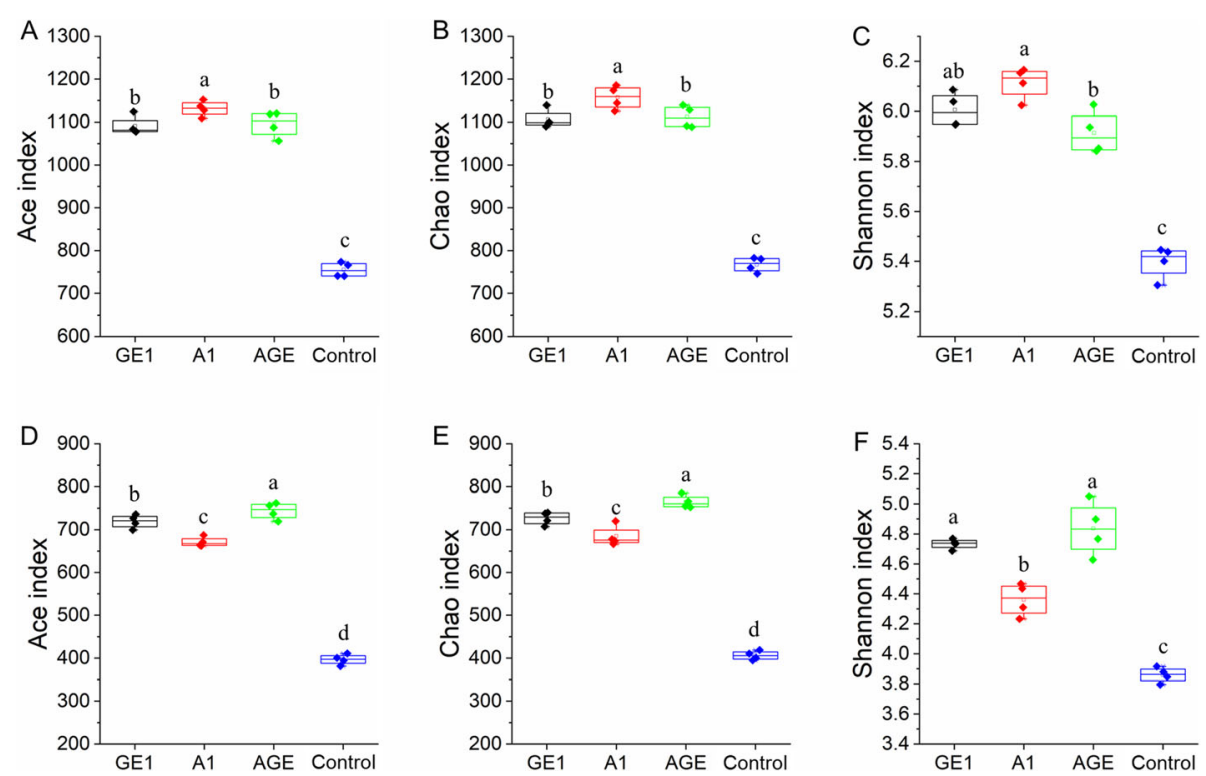

Fig. 2 Diversity indices in different rhizoshere and mycorrhizoshere soil samples. A, B, and $\mathbf{C}$ represent the Ace, Chao, and Shannon indices of bacterial community, respectively; $\mathbf{D}, \mathbf{E}$, and $\mathbf{F}$ represent the Ace, Chao, and Shannon indices of fungal community, respectively. GE1, A1, AGE, and control represent the rhizoshere or mycorrhizoshere soil of G. elata tubers, A. mellea rhizomorphs, G. elata tubers with A. mellea rhizomorphs, and unplanted, respectively. Lowercases $\mathrm{a}, \mathrm{b}, \mathrm{c}$ and $\mathrm{d}$ in figures show significantly differences between the different rhizocompartment soils (GE1, A1, AGE and control), determined by Tukey's test $(p<0.05)$

analysis (PCoA) and Hierarchical clustering analysis (HCA) based on the weighted UniFrac metric (WUF) algorithm and the Bray-Curtis distance (BCD) algorithm, respectively (Fig. 3). PCoA indicated that the microbiota community was separated by the sites of rhizoshere in G. elata cropping process, apart from GE1 and AGE. The first two axes PCoA1 and PCoA2 accounted for 62.59 and $22.33 \%$ of total variation in V3V4 (Fig. 3a), as well as 57.28 and $26.6 \%$ of total variation in ITS (Fig. $3 \mathrm{c})$, respectively. And HCA clustering also had the similar results (Fig. 3b, d). Furthermore, venn diagram analysis showed that the numbers of peculiar OTU in A1, AGE, GE1, and control groups were much higher than that of shared OTU in paired comparison analysis. But the numbers of shared OTU in AGE and GE1 showed no significant difference from the numbers of peculiar OTU in AGE or GE1 (Fig. 1B, D).

\section{Alternation of microbiota community structure under $G$. elata cropping process}

In the total V3V4 rDNA amplicons, almost OTUs (98.9-98.1\%) in all soil categories were classified as ten mains phylum, including Verrucomicrobia (1.6-2.6\%), Proteobacteria(30.0-41.1\%), Planctomycetes $(0.7-2.1 \%)$, Nitrospirae $(0.2-2.1 \%)$, Gemmatimonadetes $(0.3-2.5 \%)$, Firmicutes (0.3-2.5\%), Chloroflexi (4.0-16.7\%), Bacteroidetes (0.8-3.1\%), Actinobacteria (21.2-28.1\%), and Acidobacteria (13.6-19.4\%) (Fig. 4a). However, obvious variation trends at the phylum level were observed among the A1, GE1, AGE, and control (Fig. 4a). Compared to control, the relative abundance of Firmicutes and Actinobacteria were significantly decreased, while the relative abundance of Chloroflexi, Proteobacteria, Planctomycetes, and Gemmatimonadetes were significantly increased in GE1. However, the variation trends of Firmicutes, Chloroflexi, and Planctomycetes in A1 and AGE were the opposite (Fig. 4a). Furthermore, at the genus level, Genera with the relative abundance greater than $1 \%$ were used to evaluate and analyse the bacteria community variation, and itwas found that there was a similar variation trendscompared with the phylum level (Fig. 5).

For the fungal community, the majority OTUs (82.190.4\%) were classified as three mains phylum including Ascomycota (23.0-46.6\%), Basidiomycota (24.6-51.5\%), and Zycomycota (12.7-25.5\%). Obvious variation trends at the phylum level were also observed among the A1, GE1, AGE, and control (Fig. 4b). The relative abundance of Ascomycota was significantly increased while the relative abundance of Basidiomycota was decreased among A1, GE1, and AGE under G. elata cropping process. But, compared to control, the relative abundance of Zycomycota was increased in GE1 and AGE while decreased in A1 under G. elata cropping process (Fig. 4b). Furthermore, at the genus level, Genera with the relative abundance greater than $1 \%$ were used to evaluate and analyse the fungus community variation. The relative abundance of Trichoderma, Archaeorhizomyces, 
A
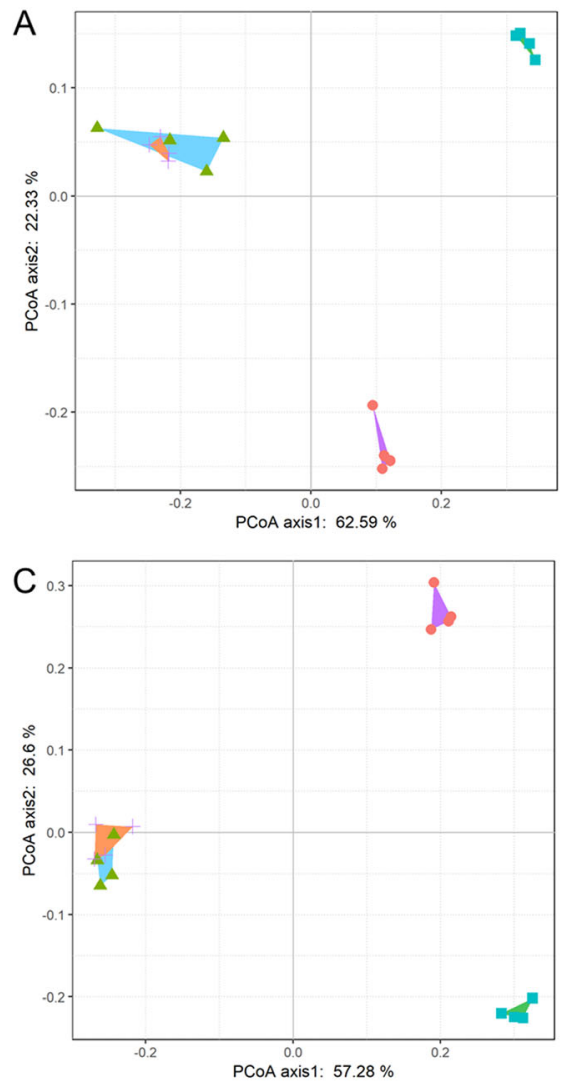

B
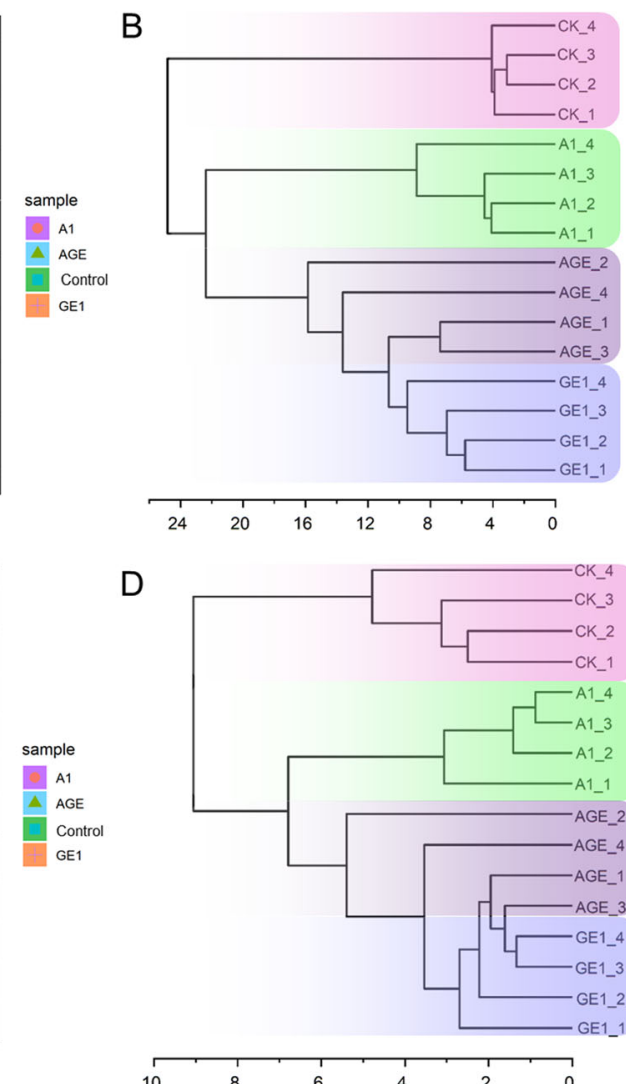

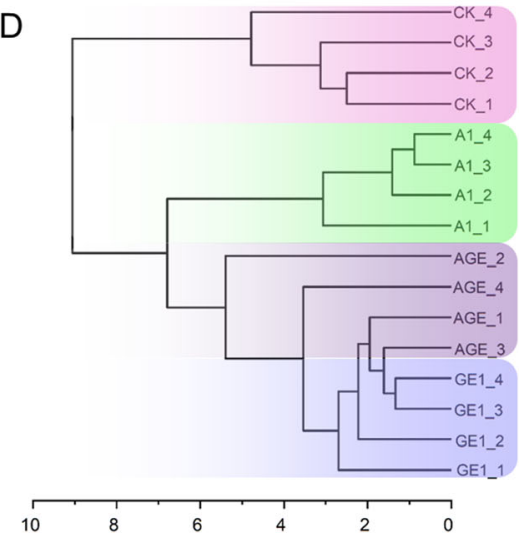

Fig. 3 Principal coordinate analysis (PCOA) based on weighted UniFrac metric (WUF) and Hierarchical clustering analysis (HCA) based on the BrayCurtis distance $(B C D)$. a and $\mathbf{b}$ represent the PCoA analysis and HCA analysis of bacterial community, respectively; $\mathbf{c}$ and $\mathbf{d}$ represent the PCoA analysis and HCA analysis of fungal community, respectively. GE1, A1, AGE, and control represent the rhizoshere or mycorrhizoshere soil of $G$. elata tubers, A. mellea rhizomorphs, G. elata tubers with A. mellea rhizomorphs, and unplanted, respectively

Metarhizium, Penicillium, Talaromyces of Ascomycota were significantly increased, while the relative abundance of Coprinellus, Amanita, Scleroderma of Basidiomycota were significantly decreased in A1 compared with control (Fig. 6).

\section{Network analysis of microbiome shifting patterns under G. elata cropping process}

A network composed of 76 nodes and 1314 edges was constructed to describe the complex relationships among soil microbiota in G. elata cropping system (Fig. 7). The network average path length was 1.77 and the network diameter was 5.00. All 76 nodes were assigned to 9 bacterial phyla and 3 fungal phyla. Eight phyla, Ascomycota (26.48\%), Basidiomycota (19.48\%), Proteobacteria (15.58\%), Actinobacteria (11.69\%), Chloroflexi (9.09\%), Acidobacteria (6.49\%), Firmicutes (3.90\%), and Verrucomicrobia (2.60\%), accounted for $93.51 \%$ of all OTUs. The 76 genera were significant correlated with G. elata cropping process, in which genus Armillaria significantly positive interacted with genus Talaromyces, Metarhizium, and Trichoderma (Ascomycota), genus
Mycobacterium of (Actinabacteria) and genus Candidatius-Xiphinematobacter (Verrucomicrobia). And more, we found that Ascomycota fungus (19 nodes) had more complex correlation with $G$. elata cropping process than Basidiomycota (13 nodes) (Fig. 7 and S2, Table S3). Bacillus interacted with 49 genus including 25 positive interaction genus and 24 negative interaction genus (Fig. 7 and S3, Table S3), which means Bacillus had important role to shape the microbial community under the $G$. elata cropping process.

\section{Discussion}

Currently, a battery of studies showed that lots of plants including crops and medicinal plants could shape the microbial community under agriculture process. This study is the first to use culture-independent next generation sequencing techniques to demonstrate the microbial community associated with G. elata and A. mellea under ternary planting system. Like many researchers reported in wheat [2], potato [27], and R. glutinosa [6], various kinds of microbe floras including fungus of the phyla Basidiomycota and Ascomycota and bacteria of 

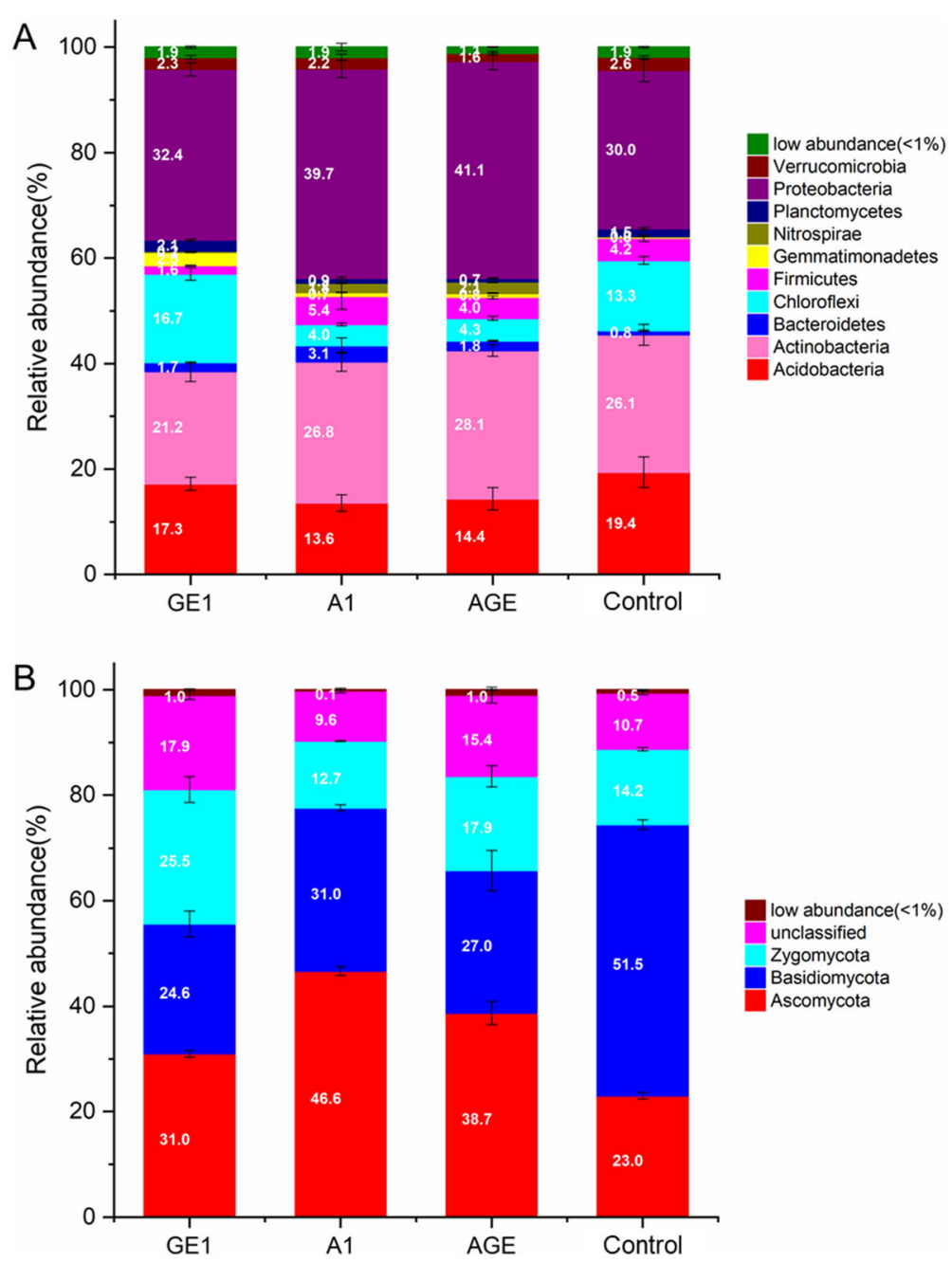

Fig. 4 The bacterial community composition (a) and fungal community composition (b) at phyla level in different rhizoshere and mycorrhizoshere soil samples. GE1, A1, AGE, and control represent the rhizoshere or mycorrhizoshere soil of G. elata tubers, A. mellea rhizomorphs, G. elata tubers with A. mellea rhizomorphs, and unplanted, respectively

the phyla Proteobacteria, Firmicutes, Chloroflexi, Actinobacteria, Acidobacteria, were significantly impacted by G. elata and A. mellea under agriculture process (Fig. 4).

Highly diverse microbial community is the important index for soil health [28], which is structurally and functionally influenced by plant and soil type [35]. As well as microbial community also can influence the plant growth, resistance to pathogens, and tolerance to stress with feedback [12]. Many previous studies showed that microbial community diversity were daftly reduced in rhizosphere soil under banana [28] and P. notoginseng [36] agriculture process. In contrast, alpha diversity analysis showed that the diversity of soil microbial communities in A. mellea mycorrhizosphere and G. elata rhizosphere were significantly increased after G. elata cropping process (Fig. 2). The reason for this contrary phenomenon may be that A. mellea as a mycorrhiza fungi can promote the growth of G. elata and increase the organic matter in mycorhizosphere soil through decaying wood materials in the ternary agriculture system [28]. Interestingly, by comparing the mycorrhizosphere soil of $A$. mellea, we found that G. elata could reduce the richness and diversity of bacterial community in rhizosphere of G. elata, but increase the richness and diversity of fungal community in rhizosphere of G. elata (Fig. 2). This results is consistent with the previously studies, in which Rehmannia glutinosa resulted in a reduction in the richness and diversity of beneficial bacteria in the monoculture regime [6], and continuous cropping of vanilla resulted in an increment in the richness and diversity of fungal pathogens [37]. The phenomenon can be explained by the selective effect of G. elata on microbe flora in rhizosphere soil, but its mechanism needs further study.

Based on the analysis of microbial community composition at the phyla level,it was found that compared 


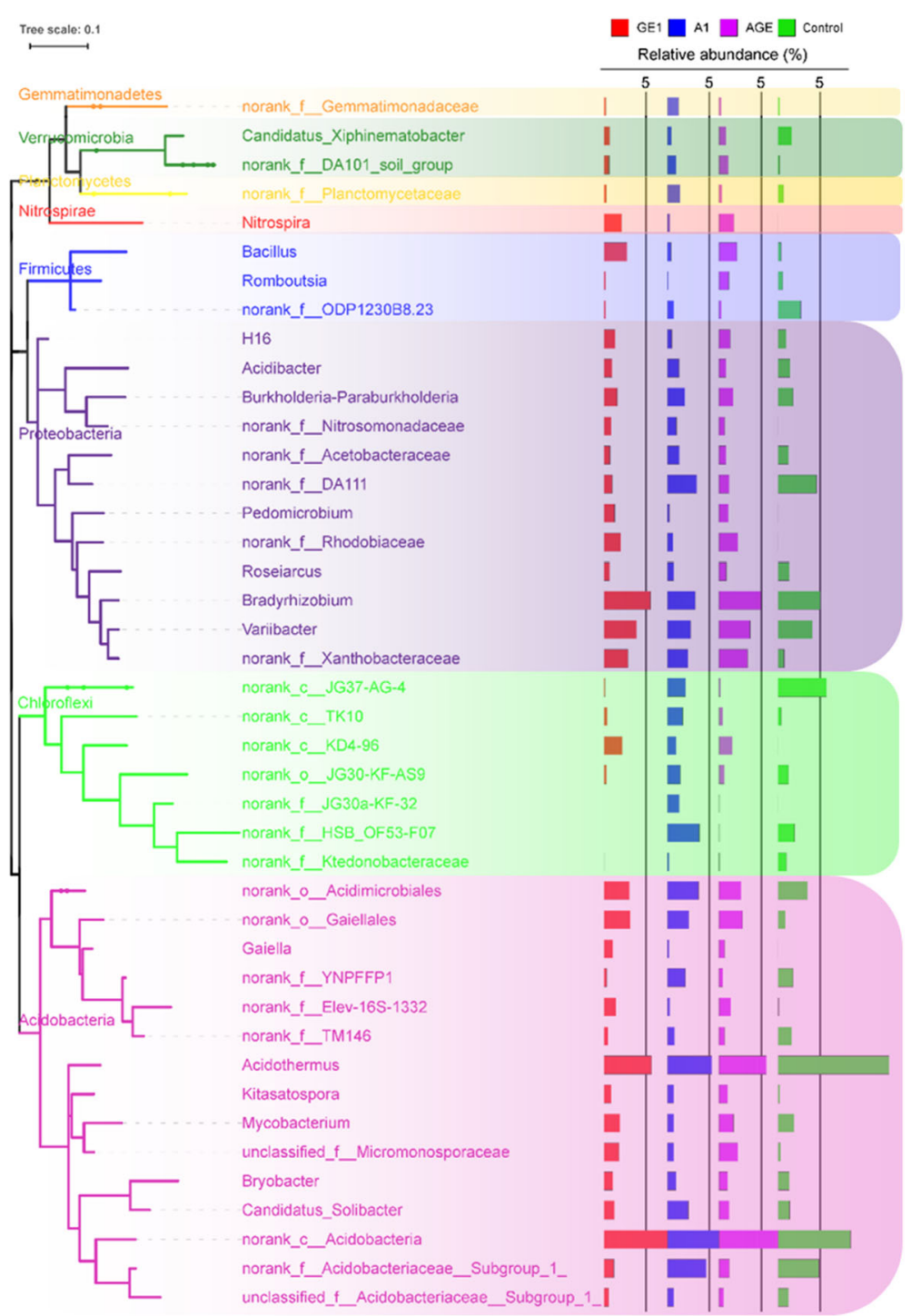

Fig. 5 Relative abundance more than $1 \%$ bacterial genus in different rhizoshere and mycorrhizoshere soil samples. GE1, A1, AGE, and control represent the rhizoshere or mycorrhizoshere soil of G. elata tubers, A. mellea rhizomorphs, G. elata tubers with A. mellea rhizomorphs, and unplanted, respectively

with control, both G. elata and A. mellea significantly decreased the relative abundance of Basidiomycete and increased the relative abundance of Ascomycete (Fig. 4). This result is in line with our study that fermentation broth of $A$. mellea significantly inhibited the growth of Phallus impudicus (data not show). Furthermore,compared with A1, GE1 and AGE significantly decreased the relative abundance of Ascomycete (genus Penicillium, Talaromyces, Leohumicola, Archaeorhizomyces, Chaetoshpaeria, Metarhizium, and Trichoderma) and Basidiomycetes (genus Cryptococcus, Russula, and Clavulinopisis) (Figs. 4, 5). This may be due to the fact that G. elata can produce antifungal proteins with strong inhibitory effects on a variety of fungus. For examples, $\mathrm{Hu}$ and Huang's research showed that gatrodia antifungal protein (GAP) or gastrodianin produced by G. elata had strongly antifungal activity against Trichoderma virde, Fusarium oxysporum and Pyricularia oryzae etc. [38]. All these results strongly indicated that the shift pattern of soil microbial communities composition in rhizosphere of G. elata tuber under agriculture process are similar to those of other medicinal plants such as $P$. heterophylla $[3,4]$ and $R$. glutinous $[5,6]$, but in mycorrhizosphere of $A$. mellea rhizomorphs is completely different.

Taxonomic analysis showed that the relative abundance of ectomycorrhizal fungus Russula, Sebacina, and Amanita decreased in mycorrhizosphere soil of $A$. 


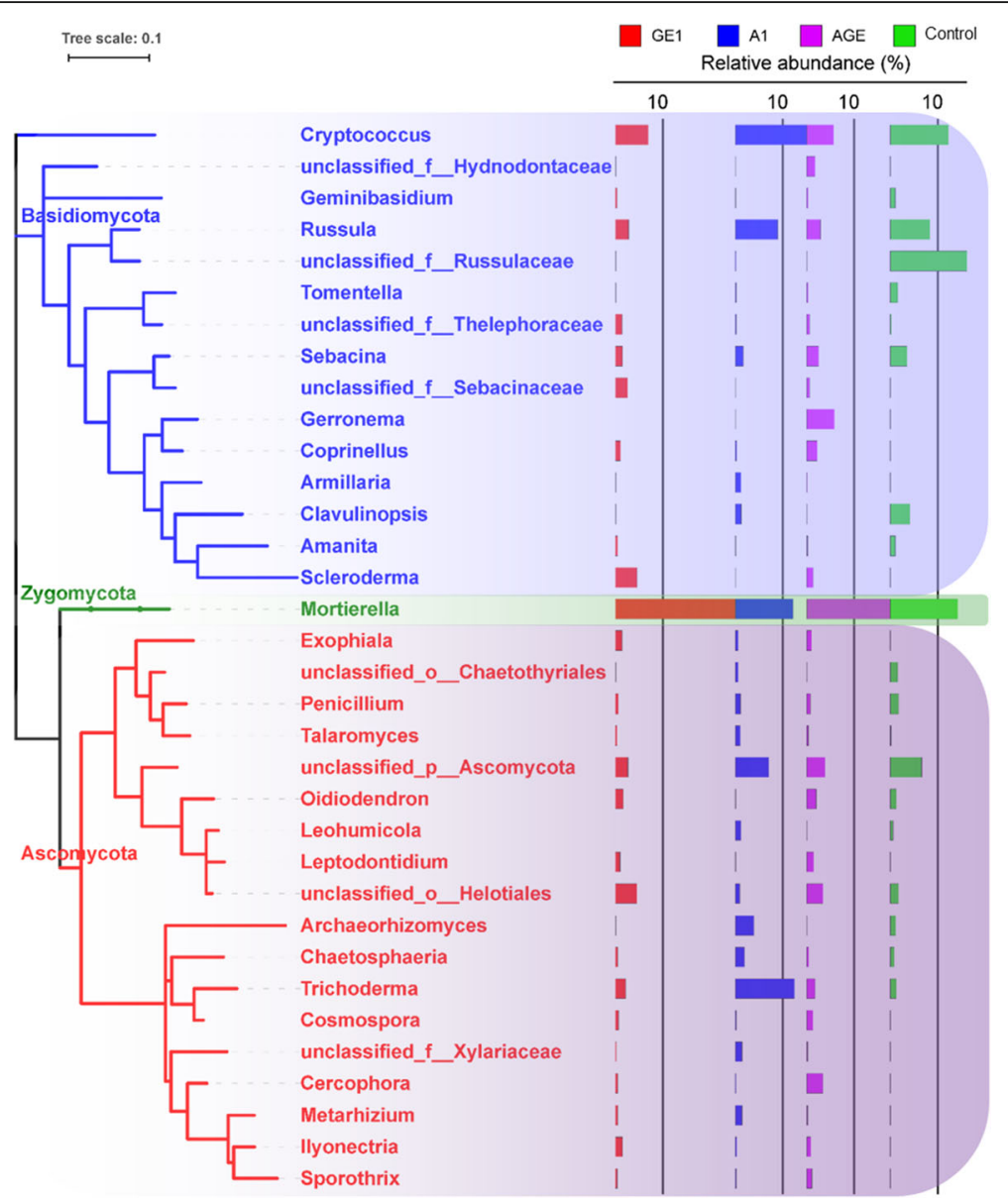

Fig. 6 Relative abundance more than 1\% fungal genus in different rhizoshere and mycorrhizoshere soil samples. GE1, A1, AGE, and control represent the rhizoshere or mycorrhizoshere soil of G. elata tubers, A. mellea rhizomorphs, G. elata tubers with A. mellea rhizomorphs, and unplanted, respectively

mellea rhizomorphs, except Scleroderma (Fig. 6). This is similar to Tuber borchii as a ectomycorrhizal fungi, which significantly reduced the abundance of other competitive mycorrhizal fungi in the early symbiotic stage with Corylus avellana [39]. On the other hand, the relative abundance of ectomycorrhizal fungus Russula, Sebacina, and Amanita also decreased in rhizosphere soil of G. elata tube, except Scleroderma (Fig. 6). Many studies have reported that ectomycorrhizal fungi such as Sebacina are significantly regulated by their host [40]. Oh et al. demonstrated that the abundance of genus Russula, Sebacina, and Amanita were highly influenced by the host plant Querus mongolica and their abundance in soil was higher than in roots of Q. mongolica [41]. Similarly, Maghnia et al. pointed out that the change of genus Russula was correlated with host Querus suber in their Northern Moroccan forest [42]. But the mechanism of how G. elata and A. mellea reduce the mycorrhizal fungi composition in (mycor) rhizoshpere needs further study.
Besides, it was found that saprophytes and pathogens such as Exophiala, Leptodontidium, Cosmospora, Cercophora, Metarhizium, Ilyonectria, and Sporothrix, were enriched in rhizoshere of G. elata and in mycorrhizosphere of $A$. mellea through taxonomic analysis (Fig. 6). This is in consistent with previous study that the fungus of Ilyonectria, a pathogen of Rusty Roots of Panax ginseng, was enriched in rhizosphere soil of Diseased-roots [36]. Similarly, Wang et al's research showed that the relative abundance of Ilyonectria in different depths apple orchard soil was increased by the application of bioorganic fertilizer through the high-throughput sequencing [43]. But, the enrichment degree of these pathogens in mycorrhizosphere of A. mellea was lower than that in rhizoshere of G. elata. This result demonstrated that $A$. mellea can reduce the enrichment of pathogens in mycorrhizosphere soil. It is coincide with Tuber borchii as ectomycorrhizal fungi similar with A. mellea, significantly decreased the abundance of pathogenic fungi during early symbiotic stage with Corylus avellana 


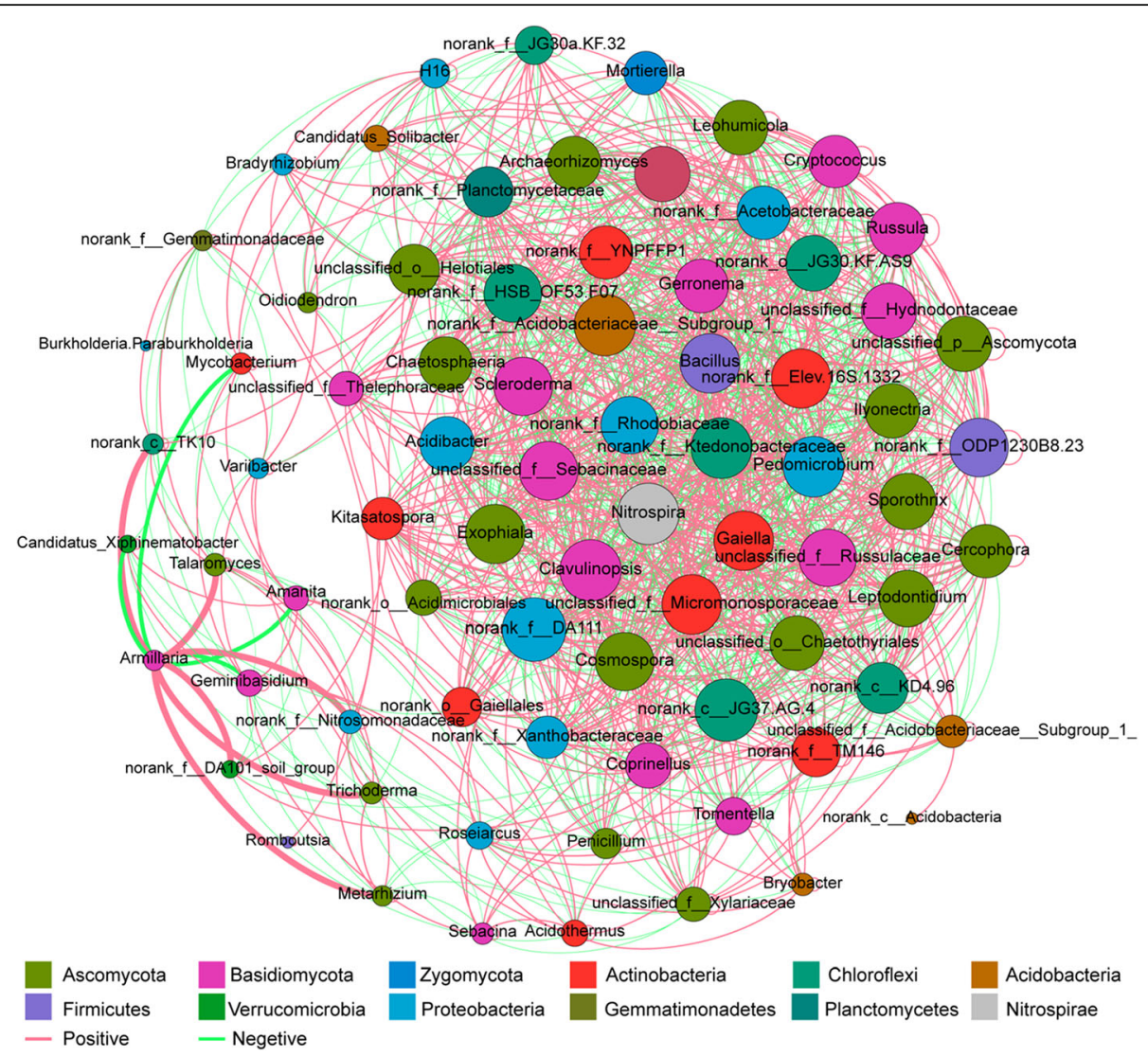

Fig. 7 Co-occurrence analysis revealed that microbial community composition reshaped under agriculture process. GE1, A1, AGE, and control represent the rhizoshere or mycorrhizoshere soil of G. elata tubers, A. mellea rhizomorphs, G. elata tubers with A. mellea rhizomorphs, and unplanted, respectively

[39]. However, the mechanism of different effects of $A$. mellea and G. elata on the saprophytes and pathogens composition in rhizoshphere needs further study.

There were research showed that Nitrospira as a kinds of chemolithoautotrophic microorganisms have the ability to oxidate ammonia via nitrite to nitrate $[44,45]$. And more, Yuan el al found that rhizomes of G. elata produced large amounts of ammonium by urease hydrolysis of urea [25]. Interestingly, the analysis of bacterial community composition at genus level showed that the relative abundance of Nitrospira, Bacillus, Pedomicrobium, norank_f_Xanthobacteraceae, and Gaiella in GE1 and AGE were higher than that in A1 and control (Fig. 6). This results showed that rhizomes of G. elata may specifically attract high nitrogen-depend bacteria such as Nitrospira, Bacillus, Pedomicrobium, norank_f Xanthobacteraceae, and Gaiella in its rhizosphere soil though ammonium production.

\section{Conclusions}

Our results showed that G. elata agriculture process clearly affected the root-associated microbial communities in both the rhizosphere and mycorrhizosphere soil. It was found that the richness and diversity of microbial communities in unplanted soil significantly increased under cropping process. In addition, the relative abundance of predominant taxa in mycorrhizosphere of A. mellea was opposite in rhizosphere of $G$. elata, including the fungal phyla (i.e., Zygomycota, Basidiomycota, and Ascomycota) and the bacterial phyla (i.e., Proteobacteria, Nitrospirae, Firmicutes, Chloroflexi, Bacteroidetes, Actinobacteria, and Acidobacteria). In particular, several members of saprophytoic and pathogenic fungus (i.e., Exophiala, Leptodontidium, Cosmospora, Cercophora, Metarhizium, Ilyonectria, and Sporothrix) were significantly enriched in mycorrhizosphere soil of A. mellea and rhizoshpere soil of G. elata, while beneficial ectomycorrhizal fungus (i.e., Russula, Sebacina, and Amanita) were significantly reduced in rhizosphere soil (Fig. 8). Further studies on maintaining the balance of soil microbiome in A. mellea mycorrhizosphere and G. elata rhizosphere under agriculture may provide a promising avenue for high yield and high quality cultivation of G. elata. 


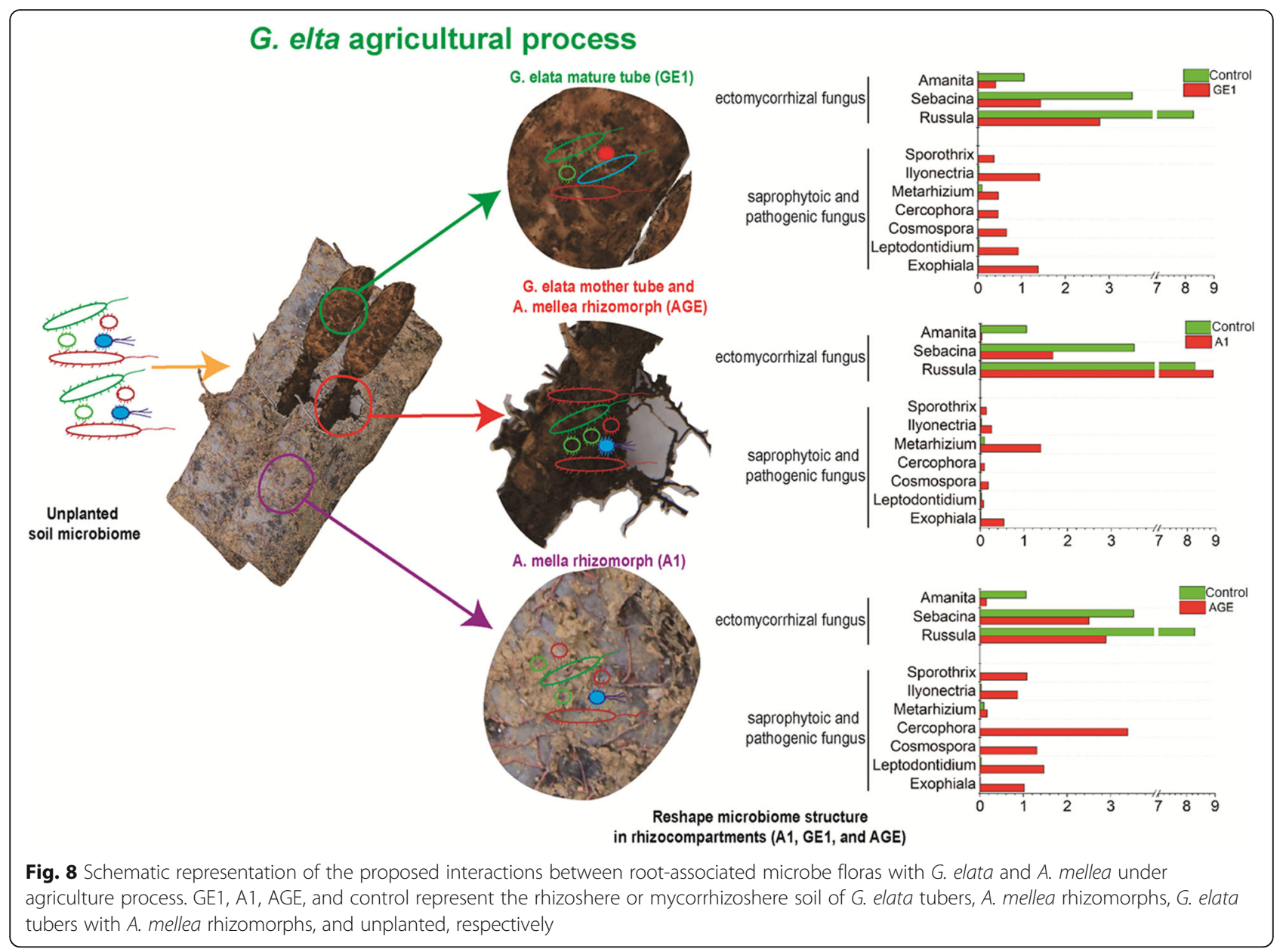

\section{Methods}

\section{Planting and sampling of Rhizosphere soil of different Partment in G. elata cropping system}

The cultivation location of G. elata was located at Dafang County, Guizhou Province, China (N27 $10^{\prime} 29^{\prime \prime}$, E105 $\left.56^{\prime} 7^{\prime \prime}\right)$ and is carried out according to WHO guidelines on good agricultural and collection practices (GACP) for medicinal plants (https://www.who.int/med icines/publications/traditional/gacp2004/en/). The mean annual temperature and precipitation in this area are $11.8^{\circ} \mathrm{C}$ and $1150 \mathrm{~mm}$, respectively. The G. elata Blume were widely planted on a large-scale in the geo-authentic areas and were selected as the experimental material. The experiment was performed in a single field site that it was newly reclaimed land and ensure the uniform growth condition among repetitions. The G. elata was cultivated as follow: first, holes with the size of $100 \mathrm{~cm}$ (length) $\times 30 \mathrm{~cm}$ (width) $\times 30-50 \mathrm{~cm}$ (depth) for each, were dug in the field; next, 5 pieces of chestnut wood $(\Phi=5-10 \mathrm{~cm}$, length $=30 \mathrm{~cm})$ fully infected by $A$. mella were put parallel on the bottom of each hole; then, $1 \mathrm{~kg}$ juvenile tubers of G. elata were put evenly on the chestnut wood, and two vials (ca. $1 \mathrm{~L}$ in total) of $A$. mella- infected twigs and leaves were crushed and sprinkled on the surface of the juvenile tubes and chestnut wood; finally, soils were filled back into the holes and covered with straw or leaves. G. elata was planted on November 12th, 2017 and sampled the rhizosphere and mycorrhizosphere soil on November 7th, 2018. G. elata of all experiments was identified by Professor Weike Jiang, and its voucher specimen was deposit on National Specimen Information Infrastructure (http://www.papc.cn/html/ folder/1-1.htm) as deposition number 000031805.

Soils from each rhizocompartment in G. elata ternary cropping system were collected (We got the permission from Guizhou Wumeng Fungi Industry Co., Ltd.), using a modification of the method previously described by [6]. In brief, G. elata mature tuber (GE1), G. elata mother tuber contained with rhizomorph of A. mellea (AGE), and rhizomorph of A. mellea (A1) were carefully uprooted and shaken to remove loosely attached soil on the GE1, AGE and A1. The GE1, AGE and A1 with tightly attached soil were added into a $50 \mathrm{~mL}$ centrifuge tube with $20 \mathrm{~mL}$ sterile water. After vortex mixing at $200 \mathrm{rpm}$ for $20 \mathrm{~min}$, the washed tubers and rhizomorph of $A$. mellea were removed and the soil suspension was 
centrifuged at $11,000 \mathrm{rpm}$ for $10 \mathrm{~min}$. The deposit was kept as the rhizosphere soil. And the unplanted field soil as the control. Each category was collected with four replications. In total, 16 samples were stored at $-70{ }^{\circ} \mathrm{C}$ for subsequent DNA extraction.

\section{DNA extract and ITS and V3V4 fragment amplification}

Microbial DNA was extracted from 16 samples using the E.Z.N.A. soil DNA Kit (Omega Bio-tech, Norcross, GA, U.S.) according to manufacturer's protocols. The final DNA concentration and purification were determined by NanoDrop 2000 UV-vis spectrophotometer (Thermo Scientific, Wilmington, USA), and DNA quality was checked by $1 \%$ agarose gel electrophoresis. The V3V4 hypervariable regions of the bacteria $16 \mathrm{~S}$ rDNA gene were selected and amplified with primers $338 \mathrm{~F}$ (5'ACTCCTACGGGAGGCAGCAG-3') and 806R (5'GGACTACHVGGGTWTCTAAT-3') by thermocycler PCR system (GeneAmp 9700, ABI, USA), as well as ITS1 regions of the fungus rDNA gene were selected and amplified with primers ITS1F (5'-CTTGGTCATT TAGAGGAAGTAA-3') and ITS2R (5'-GCTGCGTTCT TCATCGATGC- $\left.3^{\prime}\right)$. The PCR reactions were conducted by following program: pre-denaturation $3 \mathrm{~min}$ at $95^{\circ} \mathrm{C}$, 27 cycles of denaturation $30 \mathrm{~s}$ at $95^{\circ} \mathrm{C}$, annealing $30 \mathrm{~s}$ at $55^{\circ} \mathrm{C}$, and elongation $45 \mathrm{~s}$ at $72^{\circ} \mathrm{C}$, and a final extension at $72^{\circ} \mathrm{C}$ for $10 \mathrm{~min}$. PCR reactions were performed in triplicate $20 \mu \mathrm{L}$ mixture containing $4 \mu \mathrm{L}$ of $5 \times$ FastPfu Buffer, $2 \mu \mathrm{L}$ of $2.5 \mathrm{mM}$ dNTPs, $0.8 \mu \mathrm{L}$ of each primer $(5 \mu \mathrm{M}), 0.4 \mu \mathrm{L}$ of FastPfu Polymerase and $10 \mathrm{ng}$ of template DNA.

ITS and V3V4 library preparation and Illumina sequencing PCR products were isolated and extracted from a $2 \%$ agarose gel, further purified by using the AxyPrep DNA Gel Extraction Kit (Axygen Biosciences, Union City, CA, USA) and quantified using QuantiFluor ${ }^{\text {rm }}-$ ST (Promega, USA) according to the manufacturer's protocol. Purified amplicons were pooled in equimolar and paired-end sequenced $(2 \times 300)$ on an Illumina MiSeq platform (Illumina, San Diego, USA) according to the standard protocols by Majorbio Bio-Pharm Technology Co. Ltd. (Shanghai, China).

\section{Processing of sequencing data}

Trimmomatic were used to quality-filtered raw reads, and then FLASH were used to merge the paired-end reads to a tags from the high quality clean reads following criteria described by [46]. Operational taxonomic units (OTUs) were clustered with $97 \%$ similarity cutoff using UPARSE software (version 7.1, http://drive5.com/ uparse/) with a novel greedy algorithm that performs chimera filtering and OTU clustering simultaneously. The taxonomy of each V3V4 16S rDNA gene sequence was analyzed by Ribosomal Database Project Classifier algorithm (RDP, V.2.2, http://rdp.cme.msu.edu/) against the Silva (SSU123) 16S rDNA database with 70\%confidence threshold, as well as the taxonomy of each ITS1 rDNA gene sequence was analyzed by RDP algorithm against the UNITE database (Version6, Vanemuise, Tartu, Estonia).

\section{Bioinformatics analysis of microbiome sequencing}

Alpha diversity indies were used to evaluate the richness and species diversity for each sample, including observed species, Chao1, ACE, and Shannon and Simpson indexes, which were calculated by Quantitative Insights into Microbial Ecology platform (QIIME, v1.80, Twin Cities, MN, USA, 2017) based on the normalized data. Principal coordinate analysis (PCoA) based on weighted UniFrac distances (WUD) method and Hierarchical clustering analysis (HCA) based on the Bray-Curtis distance (BCD) method were performed to investigate betadiversity patterns between complex microbiota communities. Venn diagrams were constructed to visualize shared and unique OTUs among samples. The phylogenetic trees analysis of the relative abundance more than $1 \%$ genus were conducted by Molecular Evolutionary Genetics Analysis platform (MEGA, V7.0.14, https:// www.megasoftware.net/) based on the Neighbor-Joining methods. And the compositions and variation of mycrobiota community were calculated in the interactive tree of life platform based on the relative abundance of genus more than 1\% (iTOL, https://itol.embl.de/login.cgi?log out $=1$ ). The co-occurrence network analysis of the relative abundance more than $1 \%$ genus were calculated by the "psych" package in R software (V3.6.2, https://www. r-project.org/) based on the pairwise spearman method [47], and the filter conditions were significance level with cutoff value $p<0.05$ and correlation coefficient with threshold value $r>0.7$, finally, the co-occurrence diagrams were visualized by Gephi software (V0.9.2, https:// gephi.org/).

\section{Statistical analysis of sequencing data}

One-way analysis of variance (ANOVA) based on the Tukey's test method ( $\mathrm{p}<0.05, n=4)$ was carried out by SAS software (V 9.4, https://www.sas.com/en_us/) for multiple comparisons of the relative abundance of the microbiota groups and alpha diversity indices.

\section{Supplementary information}

Supplementary information accompanies this paper at https://doi.org/10. 1186/s12866-020-01790-y.

Additional file 1: Figure S1. Simposon index of bacterial (A) and fungal (B) communities in different rhizoshere and mycorrhizoshere soil samples. 
Additional file 2: Figure S2. Co-occurrence analysis revealed that bacteria community composition reshaped under agriculture process.

Additional file 3: Figure S3. Co-occurrence analysis revealed that fungi community composition reshaped under agriculture process.

Additional file 4: Table S1. Summary for bacteria V3V4 pyrosequencing and assembly.

Additional file 5: Table S2. Summary for fungal ITS pyrosequencing and assembly.

Additional file 6: Table S3. The relationship of changed microbiome at different compartment rhizoshphere at genus level.

\section{Abbreviations}

ITS: Internal transcribed spacer; V3V4: The 3rd and 4th variable regions of $16 \mathrm{~S}$ rDNA gene; NGS: Next generation sequencing; OTUs: Operational taxonomic units

\section{Acknowledgments}

We thank Guizhou Wumeng Fungi Industry Co., Ltd. to supply the fields planting G. elata. The authors would like to thank the editor and anonymous reviewers for their constructive comments that improved the earlier version of this manuscript.

\section{Authors' contributions}

QSY and JX conceived and designed the experiments; QSY and JX performed the experiments; $X O, H W$, and CX analyzed the data; YW, CK, XW, and LG contributed reagents/materials/analysis tools; QSY, WJ, and TZ wrote the paper. All authors have read and approved the manuscript.

\section{Funding}

In this work, the Key Project at Central Government Level [grant numbers 2060302] have supported for ITS and V3V4 sequencing; the National Natural Science Foundation of China [grant numbers 81960694] have supported for buy G. eleta juvenile tuber; National Key R\&D Program of China [grant numbers 2017YFC700703] have supported for G. eleta plant experiment; National Technical System of Traditional Chinese Medicine Industry [grant numbers CARS-21] have supported for G. eleta planting base rental fee; the High-level Innovative Talents of Guizhou Province of China [Qian Ke He Platform and Talent [2018]5638] have supported for transportation to and from the G. eleta planting base; the Science and Technology Project in Guizhou Province of China [Qian Ke He Platform and Talent [2019]5611] have supported for cultivate A. mellea; Guizhou Education Department Innovation Group Major Research Projects [Qian Jiao He KY Zi [2018]022] have supported for graduate subsidy; Science and Technology Plan of Guizhou Province [Qian Ke He Zhi Cheng [2018]2764] have supported for publication fee.

\section{Availability of data and materials}

All data analyzed during this study are included in this published article and its supplementary information files. Additional raw data from sequencing and quality control datasets are available from the corresponding author upon reasonable request.

\section{Ethics approval and consent to participate}

Not applicable.

\section{Consent for publication}

Not applicable.

\section{Competing interests}

The authors declare that they have no competing interests.

\section{Author details}

'Guizhou University of Traditional Chinese Medicine, Dongqingnan Road, Guiyang 540025, Guizhou, China. ${ }^{2}$ National Resource Center for Chinese Materia Medica, China Academy of Chinese Medical Sciences, Beijing 100700, China. ${ }^{3}$ Shandong Analysis and Test Center, Shandong Academic of Sciences, Jinan 250014, Shandong, China.
Received: 7 March 2020 Accepted: 16 April 2020

Published online: 05 May 2020

\section{References}

1. Li C, Li X, Kong W, Ying W, Wang J. Effect of monoculture soybean on soil microbial community in the Northeast China. Plant Soil. 2010;330:423-33.

2. Hansen JC, Schillinger WF, Sullivan TS, Paulitz TC. Soil microbial biomass and Fungi reduced with canola introduced into Long-term monoculture wheat rotations. Front Microbiol. 2019;10:1488-99.

3. Zhao Y-P, Lin S, Chu L, Gao J, Azeem S, Lin W. Insight into structure dynamics of soil microbiota mediated by the richness of replanted Pseudostellaria heterophylla. Sci Rep. 2016;6:26175-83.

4. Wu L, Chen J, Wu H, Qin X, Wang J, Wu Y, Khan MU, Lin S, Xiao Z, Luo X, et al. Insights into the regulation of Rhizosphere bacterial communities by application of bio-organic fertilizer in Pseudostellaria heterophylla monoculture regime. Front Microbiol. 2016;7:1788-801.

5. Wu L, Chen J, Khan MU, Wang J, Wu H, Xiao Z, Zhang Z, Lin W. Rhizosphere fungal community dynamics associated with Rehmannia glutinosa replant disease in a consecutive monoculture regime. Phytopathology. 2018; 108(12):1493-500.

6. Wu L, Chen J, Xiao Z, Zhu X. Barcoded pyrosequencing reveals a shift in the bacterial Community in the Rhizosphere and Rhizoplane of Rehmannia glutinosa under consecutive monoculture. Int J Mol Sci. 2018;19(3):850-66

7. Sun B, Jia S, Zhang S, McLaughlin NB, Liang A, Chen X, Liu S, Zhang X. No tillage combined with crop rotation improves soil microbial community composition and metabolic activity. Environ Sci Pollut Res Int. 2016;23(7): 6472-82.

8. Calvet C, Garcia-Figueres F, Lovato P, Camprubi A. Role of the arbuscular mycorrhizal symbiosis in tolerance response against Armillaria mellea in lavender. Span J Agric Res. 2015;13(3):e1008.

9. Haichar FEZ, Christine M, Odile B, J Ignacio RC, Prosser Jl, Jérome B, Thierry H, Wafa A. Plant host habitat and root exudates shape soil bacterial community structure. ISME J. 2008;2(12):1221-30.

10. Wei W, Yang $M$, Liu Y, Huang H, Ye C, Zheng J, Guo C, Hao M, He X, Zhu S. Fertilizer $\mathrm{N}$ application rate impacts plant-soil feedback in a sanqi production system. Sci Total Environ. 2018;633:796-807.

11. Zhang LN, Wang DC, Hu Q, Dai XQ, Xie YS, Li Q, Liu HM, Guo JH. Consortium of plant growth-promoting Rhizobacteria strains suppresses sweet pepper disease by altering the Rhizosphere microbiota. Front Microbiol. 2019;10:1668-77.

12. Luo L, Guo C, Wang L, Zhang J, Deng L, Luo K, Huang H, Liu Y, Mei X, Zhu $S$, et al. Negative plant-soil feedback driven by re-assemblage of the Rhizosphere microbiome with the growth of Panax notoginseng. Front Microbiol. 2019;10:1597-609.

13. Nefzi A, Abdallah RAB, Jabnoun-Khiareddine $H$, Ammar N, Daami-Remadi M. Ability of endophytic fungi associated with Withania somnifera L. to control Fusarium crown and root rot and to promote growth in tomato. Braz J Microbiol. 2019:50(2):481-94

14. Mi C, Yang R, Rao J, Yang S, Wei F, Li O, Hu X. Unveiling of dominant fungal pathogens associated with rusty root rot of Panax notoginseng based on multiple methods. Plant Dis. 2017;101(12):2046-52.

15. Solanki MK, Yandigeri MS. Co-inoculation of different antagonists can enhance the biocontrol activity against Rhizoctonia solani in tomato. Antonie Van Leeuwenhoek. 2019;112:1633-46.

16. Zheng YK, Miao CP, Chen HH, Huang FF, Xia YM, Chen YW, Zhao LX. Endophytic fungi harbored in Panax notoginseng: diversity and potential as biological control agents against host plant pathogens of root-rot disease. J Ginseng Res. 2017;41(3):353-60.

17. Xu X, Han L, Zhao L, Chen X, Miao C, Hu L, Huang X, Chen Y, Li Y. Echinosporin antibiotics isolated from Amycolatopsis strain and their antifungal activity against root-rot pathogens of the Panax notoginseng. Folia Microbiol (Praha). 2018;64(2):171-5.

18. McCoy AG, Noel ZA, Sparks AH, Chilvers MI. 'hagis', an R package resource for Pathotype analysis of Phytophthora sojae populations causing stem and root rot of soybean. Mol Plant-Microbe Interact. 2019;0(0):1-4.

19. Gong AD, Li HP, Yuan QS, Song XS, Yao W, He WJ, Zhang JB, Liao YC Antagonistic mechanism of iturin a and plipastatin a from Bacillus amyloliquefaciens S76-3 from wheat spikes against Fusarium graminearum. PLoS One. 2015;10(2):e0116871. 
20. Khan MR, Fischer S, Egan D, Doohan FM. Biological control of Fusarium seedling blight disease of wheat and barley. Phytopathology. 2006;96(4): 386-94

21. Li B, Li Q, Xu Z, Zhang N, Shen Q, Zhang R. Responses of beneficial Bacillus amyloliquefaciens SQR9 to different soilborne fungal pathogens through the alteration of antifungal compounds production. Front Microbiol. 2014;5: 636-45.

22. Ye T, Meng X, Wang R, Zhang C, He S, Sun G, Sun X. Gastrodin alleviates cognitive dysfunction and depressive-like behaviors by inhibiting ER stress and NLRP3 Inflammasome activation in db/db mice. Int J Mol Sci. 2018; 19(12):3977-91.

23. Luo L, Kim S-W, Lee H-K, Kim I-D, Lee H, Lee J-K. Gastrodin exerts robust neuroprotection in the postischemic brain via its protective effect against $\mathrm{Zn}(2+)$-toxicity and its anti-oxidative effects in astrocytes. Anim Cells Syst. 2018;22(6):429-37.

24. Chen L, Wang YC, Qin LY, He HY, Yu XL, Yang MZ, Zhang HB. Dynamics of fungal communities during Gastrodia elata growth. BMC Microbiol. 2019; 19(1):158-68.

25. Yuan $Y$, Jin X, Liu J, Zhao X, Zhou J, Wang X, Wang D, Lai C, Xu W, Huang J, et al. The Gastrodia elata genome provides insights into plant adaptation to heterotrophy. Nat Commun. 2018;9(1):1615-26.

26. Florent M, Maguy D, Thierry P, Paola B, Antonella F, Jacques F, Marie-Pierre $D$, Marc-André S. Independent recruitment of saprotrophic fungi as mycorrhizal partners by tropical achlorophyllous orchids. New Phytol. 2010; 184(3):668-81.

27. Li H, Wang J, Liu Q, Zhou Z, Chen F, Xiang D. Effects of consecutive monoculture of sweet potato on soil bacterial community as determined by pyrosequencing. J Basic Microbiol. 2019;59(2):181-91.

28. Shen Z, Penton CR, LV N, Xue C, Yuan X, Ruan Y, Li R. Banana Fusarium wilt disease incidence is influenced by shifts of soil microbial communities under different monoculture spans. Microb Ecol. 2018;75(3):739-50.

29. Donnelly DM, Abe F, Coveney D, Fukuda N, O'Reilly J, Polonsky J, Prange T. Antibacterial sesquiterpene aryl esters from Armillaria mellea. J Nat Prod. 1985;48(1):10-6.

30. Richard C. Antibiotic activity of Armillaria mellea. Can J Microbiol. 1971; 17(11):1395-406.

31. Obuchi T, Kondoh H, Watanabe N, Tamai M, Omura S, Yang JS, Liang XT. Armillaric acid, a new antibiotic produced by Armillaria mellea. Planta Med. 1990;56(2):198-201.

32. Ohr HD, Munnecke DE. Effects of methyl bromide on antibiotic production by Armillaria mellea. Trans Br Mycol Soc. 1974;62(1):65-74.

33. Perazzolli M, Bampi F, Faccin S, Moser M, De LF, Ciccotti AM, Velasco R, Gessler C, Pertot I, Moser C. Armillaria mellea induces a set of defense genes in grapevine roots and one of them codifies a protein with antifungal activity. Mol Plant-Microbe Interact. 2010;23(4):485-96.

34. Vitecek J, Kasparovsky T, Mikesova M, Mikes V. Nonspecific elicitation of defense reaction in suspension tobacco cells by elicitors from Armillaria. Folia Microbiol (Praha). 2005;50(2):128-32.

35. Yurgel SN, Nearing JT, Douglas GM, Langille MGI. Metagenomic functional shifts to plant induced environmental changes. Front Microbiol. 2019;10: 1682-93.

36. Liu $D$, Sun $H, M a H$. Deciphering microbiome related to rusty roots of Panax ginseng and evaluation of antagonists against pathogenic llyonectria. Front Microbiol. 2019;10:1350-61.

37. Xiong W, Zhao Q, Zhao J, Xun W, Li R, Zhang R, Wu H, Shen Q. Different continuous cropping spans significantly affect microbial community membership and structure in a vanilla-grown soil as revealed by deep pyrosequencing. Microb Ecol. 2015;70(1):209-18.

38. Hu Z, Huang QZ. Induction and accumulation of the antifungal protein in Gastrodia elata. Acta Bot Yunnanica. 1994;02:169-77.

39. Li X, Zhang X, Yang M, Yan L, Kang Z, Xiao Y, Tang P, Ye L, Zhang B, Zou J, et al. Tuber borchii shapes the Ectomycorrhizosphere microbial communities of Corylus avellana. Mycobiology. 2019;47(2):180-90

40. Long D, Liu J, Han Q, Wang X, Huang J. Ectomycorrhizal fungal communities associated with Populus simonii and Pinus tabuliformis in the hilly-gully region of the loess plateau, China. Sci Rep. 2016;6:24336-45.

41. Yang B, He F, Zhao X, Wang H, Xu X, He X, Zhu Y. Composition and function of soil fungal community during the establishment of Quercus acutissima (Carruth.) seedlings in a Cd-contaminated soil. J Environ Manag. 2019:246:150-6.
42. Maghnia FZ, Abbas Y, Mahe F, Kerdouh B, Tournier E, Ouadji M, Tisseyre P, Prin Y, El Ghachtouli N, Bakkali Yakhlef SE, et al. Habitat- and soil-related drivers of the root-associated fungal community of Quercus suber in the northern Moroccan forest. PLoS One. 2017;12(11):e0187758.

43. Wang L, Yang F, E Y, Yuan J, Raza W, Huang Q, Shen Q. Long-term application of bioorganic fertilizers improved soil biochemical properties and microbial communities of an apple orchard soil. Front Microbiol. 2016;7 1893-904.

44. Daims H, Lebedeva EV, Pjevac P, Han P, Herbold C, Albertsen M, Jehmlich N, Palatinszky M, Vierheilig J, Bulaev A, et al. Complete nitrification by Nitrospira bacteria. Nature. 2015:528(7583):504-9.

45. Pjevac P, Schauberger C, Poghosyan L, Herbold CW, van Kessel M, Daebeler A, Steinberger M, Jetten MSM, Lucker S, Wagner M, et al. AmoA-targeted polymerase chain reaction primers for the specific detection and quantification of Comammox Nitrospira in the environment. Front Microbiol. 2017;8:1508-18.

46. Yuan QS, Yang P, Wu AB, Zuo DY, He WJ, Guo MW, Huang T, Li HP, Liao YC. Variation in the microbiome, Trichothecenes, and Aflatoxins in stored wheat grains in Wuhan, China. Toxins. 2018;10(5):171-84.

47. Yan MM, Chen SN, Huang TL, Li BQ, Li N, Liu KW, Zong RR, Miao YT, Huang $X$. Community Compositions of Phytoplankton and Eukaryotes during the Mixing Periods of a Drinking Water Reservoir: Dynamics and Interactions. Int J Environ Res Public Health. 2020;17(4):1128-55. https://doi.org/10.3390/ ijerph17041128.

\section{Publisher's Note}

Springer Nature remains neutral with regard to jurisdictional claims in published maps and institutional affiliations.

Ready to submit your research? Choose BMC and benefit from:

- fast, convenient online submission

- thorough peer review by experienced researchers in your field

- rapid publication on acceptance

- support for research data, including large and complex data types

- gold Open Access which fosters wider collaboration and increased citations

- maximum visibility for your research: over $100 \mathrm{M}$ website views per year

At BMC, research is always in progress.

Learn more biomedcentral.com/submissions 